O21 (continued)

(252) most frequently with vegetables being least frequently self-served (172).

Results: Parents verbally communicated with children about food and nutrition and applied more Child Centered Nutrition Phrases when they were given education and guidance.

Conclusions and Implications: Preliminary results support education to reinforce adults verbal communication about nutrition, however continued study of adult use of CCNPs in home and child care settings is needed.

Funding: Clinical Translational Research Infrastructure Network.

\section{What Flavoring Ingredients do Low-Income Hispanic and Non-Hispanic Individuals Commonly Have?}

Het Desai, BS, het@scarletmail.rutgers.edu, Rutgers University, Snap-Ed/ EFNEP, 2nd Floor, 11 Suydam Street, New Brunswick, NJ 08901; D. Palmer, PhD

Objective: This study's objective was to compare the flavoring ingredients (i.e., herbs, spices, bouillons, salts and blends) available in low-income Hispanic and nonHispanic households.

Design, Setting and Participants: This was a descriptive, closed-ended survey study. Participants were a convenience sample of low-income individuals $(n=182)$, mostly women $(n=141)$, recruited from and interviewed at, job training and GED programs, substance abuse recovery and family success centers, and houses of worship throughout NJ. Outcome Measures and Analysis: Frequencies were calculated for demographic data, and the flavoring ingredients the participants "never," "sometimes" or "always" owned, with regards to the 34 flavoring ingredients assessed. Chi-squares and t-tests were used to compare the flavoring ingredients present in Hispanic and non-Hispanic households.

Results: Significant differences were found between the mean numbers of flavoring ingredients Hispanic $(13.7+7.3)$ vs. non-Hispanic participants $(10.8+6.0)$ kept on hand. At least $75 \%$ of all participants had both salt and garlic, while at least three-quarters of non-Hispanics also had black pepper vs. Hispanics who had cinnamon and adobo. Over 90\% of both populations "never" or "rarely" had five-spice powder, sesame seeds or dill; while non-Hispanics also rarely had mint, hot peppers, cumin or cloves. There were significant $(p>0.05)$ associations between ethnicity and "sometimes" or "always" having particular flavoring ingredients on hand, with Hispanics more often having 12 of the flavoring ingredients and non-Hispanics more often having two others.

Conclusions and Implications: The flavoring ingredients found in low-income Hispanic and non-Hispanic households appear to differ by both type and number. Nutrition educators should be aware of these differences when developing recipes for their use.

Funding: Supplemental Nutrition Assistance Program Education.
023 Cooking With Kids in Rural Minnesota: Family Meals and Interest in Family-Focused, Community-Based, Healthful-Eating Programs Sarah Friend, MPH, RD, adki0032@umn.edu, University of Minnesota, 5-140 Weaver-Densford Hall, 308 Harvard Street, Minneapolis, MN 55455; J. Fulkerson, PhD; C. Flattum, MS, RD; M. Horning, BSN, PHN; C. Olson, MEd; T. Barlow, MPH; D. Neumark-Sztainer, PhD, RD, MPH

Objective: Family meals are positively associated with healthier eating and potentially lower risk for childhood obesity. This study describes family meals among rural parents and assesses interest and barriers to attending a program similar to HOME Plus, a family-focused, communitybased, family meals program, evaluated in an urban setting as part of a clinical trial.

Design, Setting and Participants: Parents of an 8-12 year-old child with a rural zip code based on Rural Urban Commuting Access Codes (RUCA) completed a brief online survey at the Minnesota State Fair. Parents $(n=174)$ represented all three rural RUCA codes (isolated, small and large rural), had a mean age of $41(\mathrm{SD}=6.1)$ and were primarily white (89\%) and mothers (81\%).

Outcome Measures and Analysis: Chi-square tests and general linear models examined relationships between RUCA levels and family meal and program variables. Open-ended questions about healthful eating were summarized.

Results: Parents reported frequent family meals and cooking with their children; no significant differences by RUCA ( $\left.\mathrm{P}^{\prime} \mathrm{s}>0.60\right)$. Barriers to family meals and healthful eating included competing activities, time and cost; facilitators were meal planning, gardening and using farmers markets. Rural parents were interested in attending a family-meal focused, program, with most preferring in-person, weeknight programming with an online component.

Conclusions and Implications: Similar to urban families participating in HOME Plus, rural families eat frequent family meals, involve their children in meal preparation, and are interested and likely to attend family-focused programing on healthful eating, cooking, and family meals. Programs like HOME Plus designed for urban families may be acceptable to rural audiences with few modifications.

Funding: National Institutes of Health.

\section{Breakfast Eating Habits and Nutritional Status of Primary School Children in Rural Areas of Anambra State, Nigeria}

PatriciaUkegbu,PhD, MS, ukegbu.patricia@mouau.edu.ng, Michael Okpara University of Agriculture, Umudike, Department of Human Nutrition and Dietetics, Umudike, Abia State 234, Nigeria; $U$. Charity, $P h D, R D, M S$; P. Mbah, PhD, MS; E. Ifeyinwa, BS

Objective: To describe breakfast habits and nutritional status of primary school children in rural communities in Anambra State, Nigeria. 